\title{
Letter \\ Applying logic to pulmonary artery catheter use
}

\author{
Horst E Kunig ${ }^{1}$, Michael R Pinsky² and Lothar Engelmann ${ }^{3}$
}

\author{
${ }^{1}$ PO Box 192, Saltsburg, PA 15681-0192, USA \\ ${ }^{2}$ Critical Care Medicine, Bioengineering, Cardiovascular Disease and Anesthesiology, 606 Scaife Hall, 3550 Terrace Street, Pittsburgh, PA 15261, USA \\ 3 University of Leipzig, Zentrum fuer Innere Medizin, Abteilung Intensivmedizin, Liebigstrasse 20, 04103 Leipzig Germany
}

Corresponding author: Horst E Kunig, kunig@kiski.net

Published: 7 July 2008

Critical Care 2008, 12:421 (doi:10.1186/cc6928)

This article is online at http://ccforum.com/content/12/4/421

(c) 2008 BioMed Central Ltd

See related journal club critique by Mansour et al., http://ccforum.com/content/12/1/301

Mansour and colleagues recommend not routinely using the pulmonary artery catheter to guide hemodynamic management in the intensive care unit, because the perceived benefits are largely intangible [1]. Pulmonary artery catheter monitoring of the right ventricular ejection fraction (RVef) and of the right ventricular end-diastolic volume (EDV), however, reflects powerful yet underutilized relationships that assess right ventricular performance.

Since the cardiac output equals the product of the RVef, the EDV and the heart rate, one can assess the RVef to EDV relations as direct measures of right ventricular performance. A series of RVef, EDV and heart rate combinations can give the same cardiac output (Figure 1); monitoring or targeting cardiac output alone ignores this reality. For example, in hypovolemia the EDV is low and the RVef is increased, whereas in right ventricular failure the opposite is true. Furthermore, resuscitation from hypovolemia will increase the EDV and decrease the RVef [2], while reversal of cor pulmonale will decrease the EDV and increase the RVef.

No monitoring device other than the pulmonary artery catheter can continually assess these variables. These concepts are often used in the cardiac surgery suite, but are rarely codified for diagnosis and management. Monitoring devices can improve outcome only if they are coupled to treatments that improve outcome. Applying the above logic to previously proposed treatment protocols, such as using the mixed venous oxygen saturation [3], may therefore be the first step toward defining pulmonary artery catheter utility [4].

\section{Competing interests}

The authors declare that they have no competing interests.

\section{Figure 1}

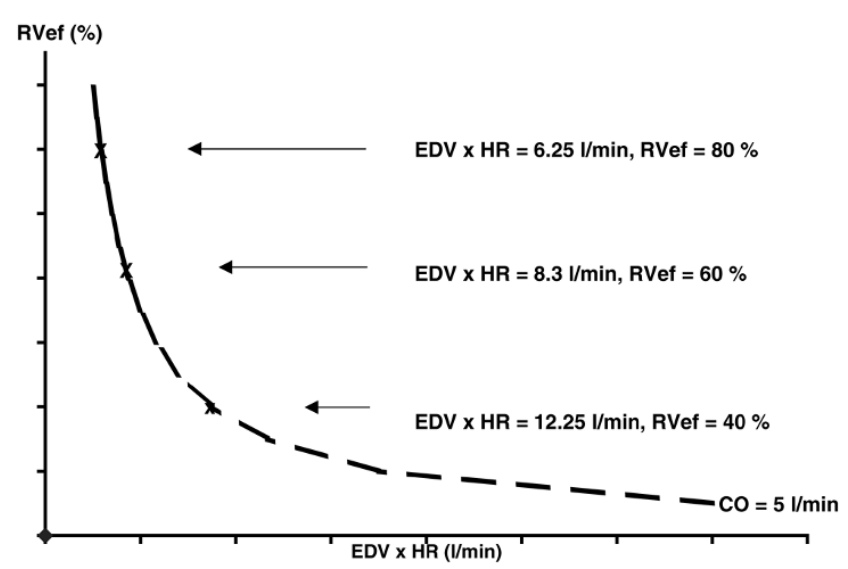

Right ventricular ejection fraction, right ventricular end-diastolic volume and heart rate relationship with cardiac output. $\mathrm{CO}$, cardiac output; EDV, right ventricular end-diastolic volume; HR, heart rate; RVef, right ventricular ejection fraction.

\section{References}

1. Mansour W, Milbrandt EB, Emlet LL: PAC in FACCT: time to PAC it in? Crit Care 2008, 12:301.

2. Dhainaut JF, Pinsky MR, Nouria S, Slomka F: Right ventricular function in human sepsis: a thermodilution study. Chest 1997 112:1043-1049.

3. Pinsky MR, Vincent J-L: Let us use the pulmonary artery catheter correctly and only when we need it. Crit Care Med 2005, 33:1119-1122.

4. Vincent JL, Pinsky MR, Sprung C, Levy M, Marini JJ, Payen D, Rhodes A, Takala J: The pulmonary artery catheter: in medio virtus. Crit Care Med (in press).

$\mathrm{EDV}=$ right ventricular end-diastolic volume; $\mathrm{RVef}=$ right ventricular ejection fraction. 